\title{
Financial Liberalization, Debt Mismatch, Allocative Efficiency and Growth
}

\author{
Romain Ranciere (PSE) and Aaron Tornell (UCLA) \\ Online Appendix
}

\section{A Model Simulations}

The behavior of the model economy is determined by seven parameters: $u, \delta, H, \alpha, \theta, \beta$, and $\mu_{w}$. We set the probability of crisis $1-u$, the degree of contract enforceability $H$, and the share of N-inputs in T-production $\alpha$ equal to empirical counterparts in emerging markets. Then, given the values of $u, H$, and $\alpha$, we set the discount factor $\delta$, productivity in the N-sector $\theta$, and N-sector internal funds $1-\beta$ such that both an RSE and an SSE exist and the central planer's optimal investment share is less than one. The value of the crisis costs $\mu_{w}$ is irrelevant for the existence of equilibria. The admissible parameter set is determined by the following conditions.

- $H<1$ : necessary for borrowing constraints to bind in the safe equilibrium.

- $u>H$ : necessary for borrowing constraints to bind in the risky equilibrium.

- $\beta>\underline{\beta}=H / u$ : necessary and sufficient for positive prices: $\phi^{l}<1, \phi^{s}<1$.

- $\delta \theta^{\alpha}<1$ : necessary and sufficient for a interior solution of the central planner's problem.

- $\theta>\underline{\theta}^{s}(\delta, H, \alpha, \beta)$ : necessary and sufficient for the SSE's rate of return on equity to be larger than the risk-free rate.

- $\theta<\bar{\theta}\left(\delta, H, u, \alpha, \mu_{w}, \beta\right)$ : necessary and sufficient for default in the low price state.

- $\theta>\underline{\theta}(\delta, H, u, \alpha, \beta)$ : necessary and sufficient for the RSE's rate of return on equity to be larger than the risk-free rate.

- $\beta<\bar{\beta}\left(\delta, H, u, \alpha, \mu_{w}\right)$ : necessary and sufficient for $\bar{\theta}>\underline{\theta}$.

The bounds $\bar{\beta}, \underline{\theta}, \bar{\theta}$ are given by (17)-(18) and $\underline{\theta}^{s} \equiv\left[\frac{1}{\beta \delta}\right]^{\frac{1}{\alpha}}\left[\frac{1-\beta}{1-H}\right]^{\frac{1-\alpha}{\alpha}}$.

In the simulations, the baseline crisis probability is set to $5 \%$, which is (i) slightly higher than the unconditional crisis probability in Schularick and Taylor (4.49\%) and Gourinchas and Obstfeld (2.8\%), and (ii) close to the 75th percentile for the predicted crisis probability in these two papers. We calibrate $H$ in reference to the debt-to-assets ratio estimated in Section 4 for 
the sample emerging markets over 1994-2013. The debt-to-assets ratio is 0.5425 which combined with a crisis probability of $5 \%$ yields: $H=u \frac{\text { debt }}{\text { assets }}=0.515$. We set $\alpha=0.34$, which is calibrated in reference to the average use of non-tradable goods as inputs in tradeables production across 7 countries in Emerging Asia. ${ }^{50}$ The parameters $\delta$ and $\theta$ are chosen so as to satisfy the parametric restrictions for existence of an RSE over the range $u \in(0.9,1)$, while delivering plausible values for the growth rates along a safe equilibrium and along a tranquil path of an RSE. We thus set $\theta=1.6$ and $1-\beta=0.33$, which imply a safe GDP growth rate of $\left(1+\gamma^{s}\right)=(1-\beta)^{\alpha}\left(\frac{\theta}{1-H}\right)^{\alpha}=2.57 \%$ and a tranquil times GDP growth rate of $\left(1+\gamma^{l}\right)=(1-\beta)^{\alpha}\left(\frac{\theta}{1-H u^{-1}}\right)^{\alpha}=4.59 \%$. We choose a lower bound for the financial distress costs of crises $l^{d}=1-\frac{\mu_{w}}{1-\beta}$ so that the severity of crisis, derived in Section 4, matches the annualized average GDP loss (10.68\%) in Laeven and Valencia (2012)..$^{51}$ The upper bound for $l^{d}$ is set to $76.6 \%$, which is above the threshold for gains from financial liberalization (65.40\%). Finally, the choice of the discount factor has no impact on the growth rates of the decentralized economy. The discount factor is set to $\delta=0.85$ to satisfy $\delta<\theta^{-\alpha}$ so that $\phi^{c p}<1$. Summing up:

\begin{tabular}{|c|c|c|c|}
\hline Parameters & $\begin{array}{c}\text { baseline } \\
\text { value }\end{array}$ & $\begin{array}{l}\text { range of } \\
\text { variation }\end{array}$ & target / sources \\
\hline Probability of crisis & $1-u=0.05$ & {$[0,0.1]$} & $\begin{array}{c}\text { Schularick-Taylor (2012), } \\
\text { Gourinchas-Obstbeld (2012) }\end{array}$ \\
\hline $\begin{array}{l}\text { Intensity of N-inputs } \\
\text { in T-production }\end{array}$ & $\alpha=0.34$ & {$[0.2,0.4]$} & $\begin{array}{c}\text { Input-Output Tables for Emerging Asia } \\
\text { Source: ADB (2012) }\end{array}$ \\
\hline Financial distress costs & $l^{d}=24 \%$ & {$[18 \%, 76.6 \%]$} & Laeven and Valencia (2013) \\
\hline Contract enforceability & $H=0.515$ & & $\begin{array}{l}\text { Debt-to-Assets in Emerging Countries } \\
\text { Source: Thompson Worldscope }\end{array}$ \\
\hline N-sector Internal Funds & $1-\beta=0.33$ & & \\
\hline N-sector Productivity & $\theta=1.6$ & & \\
\hline
\end{tabular}

Table A1. Calibration of the Model

\footnotetext{
${ }^{50}$ The set of countries include India, Thailand, China, Indonesia, Malaysia, Sri-Lanka and Taiwan. The source for the input-output tables used in the calculations is the Asian Development Bank (ADB, 2012).

${ }^{51}$ Formally, we back out $\mu_{w}$ by setting $S=1-\left(\frac{1-H u-1}{1-H} \cdot \frac{\mu_{w}}{1-\beta}\right)^{\alpha}=0.1068$ which. given the numerical values of the other parameters, implies $l^{d}=1-\frac{\mu_{w}}{1-\beta}=23.3 \%$
} 


\section{B Estimates of the Crisis Probability}

The literature considers both the unconditional probability - the frequency of crisis in a given sample - and the predicted probability, typically generated by a logit or probit model. We consider the samples of Schularick and Taylor (2012)[ST], who consider a long historical sample of 14 countries over the period 1870-2008, and Gourinchas and Obstfeld (2012) [GO], who consider a sample of 79 advanced and emerging economies over the period 1970-2012.

Schularick and Taylor (2012). Their sample includes: Australia, Canada, Switzerland, Germany, Denmark, Spain, France, Great Britain, Italy, Japan, Netherlands, Norway, Sweden and the USA. These countries are now developed, but most could be considered "emerging" for a good part of the sample. ST identify 79 major financial crises in their sample. This number of crises implies an unconditional probability that a crisis starts in given year of $4.49 \%$.

They estimate a logit model with five lags of credit growth to predict the probability of financial crises. The pseudo-R2 of their logit regression is $4.34 \%$ without country fixed-effects (and $6.59 \%$ when fixed effects are included), which means that the predictive power of their model is rather

limited. Another way to see this is to look at the predicted probability of crisis, which can be estimated for each country-year by using the estimated logit model. The distribution of predicted crises probabilities is summarized in the next table.

$\begin{array}{cccccc}\text { percentile of country-years } & 5 \% & 25 \% & 50 \% & 75 \% & 95 \% \\ \text { crisis probability } & 1.47 \% & 2.54 \% & 3.48 \% & 4.82 \% & 8.55 \%\end{array}$

Table A2: Predicted Crisis Probability (Schularick-Taylor, 2012)

The table reveals that for $95 \%$ of the country-years the crisis probability is less than $8.5 \%$, which implies that even when credit growth is very high, the actual occurrence of crisis remains the effect of a mostly random component. Only 5 observations (out of 1272) exhibit a crisis probability of more than 20\% (Germany (1954, 1955), Japan (1964,1972), and France (1906)), out of which only one had a crisis within the next five years (France, 1907). If we look at the crisis probability on the year just before each major crisis, the average crisis probability is $4.8 \%$. This is only slightly higher than the unconditional probability in the regression sample $(4.08 \%)$.

If we consider three subperiods with distinct regulatory regimes we find the following. During the Breton Woods era (1944-1972) — a financially repressed period with tight regulations on capital flows - there were zero crises, yet the model predicted crises with an average probability of $5.32 \%$. In the post Breton Woods era (1972-2012), the unconditional crisis probability was $3.8 \%$ and the 
predicted probability was on average $4.06 \%$. Finally, in the first era of financial liberalization (1870-1914), these probabilities were $6.03 \%$ and $4.16 \%$, respectively.

Gourinchas and Obstfeld (2012). Based on their updated appendix, the unconditional probabilities that a crisis starts in a given year are:

$\begin{array}{ccc}\text { Sample } & \text { Systemic Banking Crises } & \text { Currency Crises } \\ \text { Advanced } & 2.32 \% & 5.26 \% \\ \text { Emerging } & 2.8 \% & 4.15 \%\end{array}$

Table A3. Unconditional Crises Probabilities (1970-2010)

To complement ST's results, we focus on their predictive regression of systemic banking crises in their sample of 57 emerging markets. In contrast with ST, who focus on the role of credit, GO introduce 7 variables in their specification: Public Debt to GDP, Credit to GDP, Current Account to GDP, Reserves to GDP, Real Exchange Rate, Short Term Debt to GDP and the Output Gap. Their richer specification, and the omission of four years of variables after each crisis, allows them to increase the pseudo R-squared of their regression up to 17 percent (21 percent with fixed effects). We report below the distribution of the predicted crisis probabilities for three specification: (i) the full specification with all 7 regressors; (ii) a specification with only debt to GDP and credit to GDP, which is closer to our model, and (iii) a specification with only credit to GDP, which is closer to ST.

$\begin{array}{cccccc}\text { percentile of country-years } & 5 \% & 25 \% & 50 \% & 75 \% & 95 \% \\ \begin{array}{c}\text { crisis probability } \\ \text { full specification }\end{array} & 0.37 \% & 1.47 \% & 2.96 \% & 5.7 \% & 17.74 \% \\ \begin{array}{c}\text { crisis probability } \\ \text { credit/GDP }\end{array} & 1.84 \% & 2.97 \% & 3.69 \% & 4.87 \% & 7.16 \% \\ \begin{array}{c}\text { Short Term Debt/GDP } \\ \text { crisis probability } \\ \text { credit/GDP only }\end{array} & 1.8 \% & 2.91 \% & 3.57 \% & 4.44 \% & 7.76 \%\end{array}$

Table A4: Predicted Crisis Probability (Gourinchas and Obstfeld, 2012)

The distribution of predicted crises probabilities displays a similar pattern as that in ST. Especially so in the last two specifications. Even in the richest specification, $95 \%$ of the observations 
display a crisis probability of less than $18 \%$. Only 3 (out of 812 ) country-years have a crisis probability higher than 0.512 , which is the maximum admissible probability of crisis consistent with our estimate of $H$ (0.488). In the second specification, which is closer to our model, only 7 observations (out of 1224) display a crisis probability higher than $30 \%$.

\section{Proofs and Derivations.}

Derivation of (12). Any solution to the central planner's problem is characterized by the optimal accumulation of $\mathrm{N}$-goods that maximizes the discounted sum of T-production

$$
\max _{\left\{d_{t}\right\} \in \mathcal{C}^{1}} \sum_{t=0}^{\infty} \delta^{t} d_{t}^{\alpha}, \quad \text { s.t. } \quad k_{t+1}=\left\{\begin{array}{cc}
\theta k_{t}-d_{t} & \text { if } t \geq 1 \\
q_{0}-d_{0} & \text { if } t=0
\end{array}, \quad d_{t} \geq 0, q_{0}\right. \text { given }
$$

The Hamiltonian associated with this problem is $H_{t}=\delta^{t}\left[d_{t}\right]^{\alpha}+\lambda_{t}\left[\theta k_{t}-d_{t}\right]$. Since $\alpha \in(0,1)$, the necessary and sufficient conditions for an optimum are

$$
0=H_{d}=\delta^{t} \alpha\left[d_{t}\right]^{\alpha-1}-\lambda_{t}, \quad \lambda_{t-1}=H_{k}=\theta \lambda_{t}, \quad \lim _{t \rightarrow \infty} \lambda_{t} k_{t}=0
$$

Thus, the Euler equation is

$$
d_{t+1}=[\delta \theta]^{\frac{1}{1-\alpha}} d_{t}=\theta \hat{\phi} d_{t}, \quad \hat{\phi} \equiv\left[\delta \theta^{\alpha}\right]^{\frac{1}{1-\alpha}} \quad t \geq 1
$$

To get a closed form solution for $d_{t}$ we replace (48) in the accumulation equation:

$$
k_{t}=\theta^{t-1} k_{1}-d_{0} \sum_{s=0}^{t-2} \theta^{t-s-2}[\delta \theta]^{\frac{s+1}{1-\alpha}}=\theta^{t-1}\left[k_{1}-d_{0} \hat{\phi} \frac{1-\hat{\phi}^{t-1}}{1-\hat{\phi}}\right]=\theta^{t-1}\left[k_{1}-\frac{d_{1}}{\theta} \frac{1-\hat{\phi}^{t-1}}{1-\hat{\phi}}\right]
$$

Replacing (48) and (49) in the transversality condition we get

$$
\begin{aligned}
0 & =\lim _{t \rightarrow \infty} \delta^{t} \alpha\left[d_{t}\right]^{\alpha-1} k_{t}=\lim _{t \rightarrow \infty} \delta^{t} \alpha\left[[\delta \theta]^{\frac{t}{1-\alpha}} d_{0}\right]^{\alpha-1}\left[\theta^{t-1} k_{1}-d_{0} \hat{\phi} \frac{1-\hat{\phi}^{t-1}}{1-\hat{\phi}}\right] \\
& =\frac{\alpha d_{0}^{\alpha-1}}{\theta}\left[k_{1}-d_{0} \hat{\phi} \frac{1}{1-\hat{\phi}}\right] \Leftrightarrow \hat{\phi}<1
\end{aligned}
$$

Since $k_{1}=q_{0}-d_{0}$, the bracketed term equals zero if and only if $\hat{d}_{0}=[1-\hat{\phi}] q_{0}$. The accumulation equation then implies that the unique optimal solution is $\hat{d}_{t}=[1-\hat{\phi}] q_{t}$.

Proof of Proposition 3.1. First, we determine the conditions on returns $\frac{\beta \theta p_{t+1}^{e}}{p_{t}}$ that make the strategy of Proposition 3.1 optimal for an individual entrepreneur, given that all other entrepreneurs follow the equilibrium strategy: borrow up to the limit (i.e., $\delta b_{t} p_{t+1}\left[1+\rho_{t}\right]=H\left[w_{t}+b_{t}\right]$ ), invest all funds in the production of $\mathrm{N}$-goods $\left(p_{t} k_{t}=w_{t}+b_{t}, s_{t}=0\right)$, and never default. We then determine 
the parameter conditions under which the price sequences, that result if all entrepreneurs follow the equilibrium strategy, generate a high enough return to validate the strategy of an individual entrepreneur.

Given that all other entrepreneurs follow the equilibrium strategy, crises never occur and prices are deterministic: $u_{t+1}=1$. Thus no bailout is expected. First, since no bailout is expected, lenders will get repaid zero with any plan that leads to diversion. Hence, lenders only fund plans where the no-diversion condition holds. Second, since competitive risk-neutral lenders have to break even: the interest rate offered on $\mathrm{N}$-debt is $1+\rho^{N}=[1+r] / E_{t}\left(p_{t+1}\right)$, while that on T-debt is $\rho^{T}=r$. Thus, the expected interest costs are the same under both types of debt: $\left[1+\rho^{N}\right] E_{t}\left(p_{t+1}\right)=1+\rho^{T}=1+r$. Hence, the borrowing limits are the same under both types of debt, and so there is no incentive to issue T-debt. It follows that if all other firms choose a safe plan, the payoff of a safe plan is the solution to the following problem

$$
\begin{aligned}
\max _{b_{i, t}, k_{i, t+1}, l_{i, t+1}} Z_{i, t} & \left.=\left\{E_{t}\left(p_{t+1}\right) \Theta_{t+1} k_{i, t+1}^{\beta} l_{i, t+1}^{1-\beta}+\left[s_{i, t}-b_{i, t}\right][1+r]-v_{t+1} l_{i, t+1}\right\} \delta, \text { subject t } 650\right) \\
p_{t} k_{i, t} & \leq w_{i, t}+b_{i, t}-s_{i, t}, \quad b_{i, t} \leq H\left[w_{i, t}+b_{i, t}\right], \quad \pi_{i, t+1}^{s} \geq 0
\end{aligned}
$$

where prices and the wage are taken as given. Suppose for a moment that $\frac{\beta \theta E_{t}\left(p_{t+1}\right)}{p_{t}}$ is high enough so that it is optimal to borrow up to the limit allowed by the no-diversion condition $\left(b_{i, t}=H\left[w_{i, t}+b_{i, t}\right]\right)$, and not store (so that $\left.p_{t} k_{i, t+1}=w_{i, t}+b_{i, t}\right)$. It follows that the first order conditions are

$$
\frac{\partial Z_{i, t}}{\partial k_{i, t+1}}=E_{t}\left(p_{t+1}\right) \Theta_{t+1} l_{i, t+1}^{1-\beta} k_{i, t+1}^{\beta-1} \beta \delta-H p_{t} \geq 0, \quad \frac{\partial Z_{i, t}}{\partial l_{i, t+1}}=p_{t+1} \Theta_{t+1} l_{i, t+1}^{-\beta} k_{i, t+1}^{\beta}[1-\beta]-v_{t+1} \geq 0 .
$$

Notice that $\pi_{t+1}^{s}$ is concave in $k_{i, t+1}$ because $\beta<1$. Since in a SSE all entrepreneurs choose the same investment level, $\Theta_{t+1} k_{i, t+1}^{\beta-1}=\theta \bar{k}_{t+1}^{1-\beta} k_{i, t+1}^{\beta-1}=\theta$. Furthermore, since labor is inelastically supplied $\left(l^{s}=1\right)$, the equilibrium wage is

$$
\widehat{v}_{t+1}=p_{t+1} \theta k_{t+1}[1-\beta] .
$$

Substituting (52) in (51) we have that in an SSE, the marginal return of capital is:

$$
\left.\frac{\partial Z_{i, t}}{\partial k_{i, t+1}}\right|_{\widehat{v}_{t+1}, k_{i, t+1}=\bar{k}_{t+1}}=E_{t}\left(p_{t+1}\right) \theta \beta \delta-H p_{t} .
$$

Thus, if $E_{t}\left(p_{t+1}\right) \theta \beta \delta>H p_{t}$, the solution to (50) entails borrowing and investing as much as allowed by the no-diversion condition $b_{i, t}=H\left[w_{i, t}+b_{i, t}\right]$. It follows that the payoff associated with the equilibrium strategy is

$Z_{t+1}^{s}=\delta\left\{\beta \theta p_{t+1} k_{t+1}-[1+r] b_{t}\right\}=\left[\frac{\delta \beta \theta p_{t+1}}{p_{t}}-H\right]\left[w_{t}+b_{t}\right]=\left[\frac{\delta \beta \theta p_{t+1}}{p_{t}}-H\right] m^{s} w_{t}, \quad m^{s} \equiv \frac{1}{1-H}$. 
In order for the above solution to be optimal, this return on equity must be greater than the storage return: $\left[\delta \beta \theta p_{t+1} / p_{t}-H\right] m^{s} w_{t}>w_{t}$. This condition is equivalent to $\delta \beta \theta p_{t+1} / p_{t}>1$. To determine whether this condition is satisfied we need to endogeneize prices. To do so we use $q_{t}=\theta \phi_{t-1} q_{t-1}$ and $p_{t}=\alpha\left[q_{t}\left(1-\phi_{t}\right)\right]^{\alpha-1}$, and find that in a SSE $\frac{p_{t+1}}{p_{t}}=\left(\theta \phi^{s}\right)^{\alpha-1}$, with $\phi^{s}=\frac{1-\beta}{1-H}$. Therefore,

$$
\frac{\beta \theta p_{t+1}}{p_{t}}>\frac{1}{\delta} \Leftrightarrow \beta \theta^{\alpha}\left(\phi^{s}\right)^{\alpha-1}>\frac{1}{\delta} \Leftrightarrow \theta>\underline{\theta}^{s} \equiv\left[\frac{1}{\beta \delta}\right]^{\frac{1}{\alpha}}\left[\frac{1-\beta}{1-H}\right]^{\frac{1-\alpha}{\alpha}} .
$$

We conclude that the SSE characterized in Proposition 3.1 exists if and only if (54) holds, and $\beta<\bar{\beta}$ so that prices are positive (i.e., $\phi^{s}<1$ ). Finally, notice that, because there is a production externality, there exists also a degenerate equilibrium in which nobody invests because everyone believes others will not invest: $\theta \bar{k}_{t+1}^{1-\beta}=0$.

Proof of Proposition 3.2. The proof is in two parts. In part A we construct an RSE where two crises do not occur in consecutive periods. Then, in part B we show that two crises cannot occur in consecutive periods.

Part A. Consider an RSE where, if there is no crisis at $t$, prices next period can take two values as in (22). Meanwhile, if there is a crisis at $t$, there is a unique $p_{t+1}$. In a no crisis period, a firm can choose three types of plans: a "risky plan" where a firm denominates debt in T-goods (i.e., with debt denomination mismatch), will default if $p_{t+1}=\underline{p}_{t+1}$, and does not divert; a "safe plan" where a firm denominates debt in N-goods, will never default and does not divert; finally a "diversion plan" where the firm will divert all funds. We will construct an RSE in which all entrepreneurs find it optimal to choose the risky plan during every period, except when a crisis erupts, in which case they choose the safe plan.

Suppose for a moment that $\underline{p}_{t+1}$ is low enough so as to bankrupt firms with T-debt (we will determine below the parameter set under which this holds). Since in an RSE every firm issues T-debt, a bailout will be granted next period in the low price state. In this case lenders will be repaid in all states (either by the borrowers or by the bailout agency) and so they break-even if the interest rate is $\rho^{T}=r$. It follows that if all other firms choose a risky plan, the payoff of a risky plan is the solution to the following problem:

$$
\begin{aligned}
\max _{b_{i, t}, k_{i, t+1}, l_{i, t+1}} Z_{i, t+1}^{r} & =\delta u\left\{\bar{p}_{t+1} \Theta_{t+1} k_{i, t+1}^{\beta} l_{i, t+1}^{1-\beta}+\left[s_{i, t}-b_{i, t}\right][1+r]-v_{t+1} l_{i, t+1}\right\}, \text { subject to }(55) \\
p_{t} k_{i, t} & \leq w_{i, t}+b_{i, t}, \delta u[1+r] b_{i, t} \leq H\left[w_{t}+b_{i, t}\right], \quad \pi_{i, t+1}^{r}\left(\bar{p}_{t+1}\right) \geq 0, \quad \pi_{i, t+1}^{r}\left(\underline{p}_{t+1}\right)<0
\end{aligned}
$$

where $\left(p_{t}, \underline{p}_{t+1}, \bar{p}_{t+1}, v_{t+1}, \Theta_{t+1}\right)$ are taken as given. The first order conditions are

$$
\frac{\partial Z_{i, t+1}^{r}}{\partial k_{i, t+1}}=u \bar{p}_{t+1} \Theta_{t+1} l_{i, t+1}^{1-\beta} k_{i, t+1}^{\beta-1} \beta \delta-H p_{t} \geq 0, \quad \frac{\partial Z_{i, t+1}^{r}}{\partial l_{i, t+1}}=p_{t+1} \Theta_{t+1} l_{i, t+1}^{-\beta} k_{i, t+1}^{\beta}[1-\beta]-v_{t+1} \geq 0
$$


Notice that $\pi_{t+1}^{r}$ is concave in $k_{i, t+1}$ because $\beta<1$. Since in an RSE all entrepreneurs choose the same investment level, $\Theta_{t+1} k_{i, t+1}^{\beta-1}=\theta \bar{k}_{t+1}^{1-\beta} k_{i, t+1}^{\beta-1}=\theta$. Furthermore, the equilibrium wage is given by (52). Following the same steps as in the proof of Proposition 3.1, we have that if $\delta u \frac{\beta \theta \bar{p}_{t+1}}{p_{t}}>H$, the solution to (55) entails borrowing up to the limit allowed by the no-diversion constraint: $b_{t}=$ $\left[m^{r}-1\right] w_{t}=\left[m^{r} H / u\right] w_{t}$. Thus, the payoff is ${ }^{52}$

$$
Z_{t+1}^{r}=\left[\delta u \frac{\beta \theta \bar{p}_{t+1}}{p_{t}}-H\right] m^{r} w_{t}, \quad m^{r} \equiv \frac{1}{1-H / u} .
$$

In order for a firm to choose a risky plan the following conditions must be satisfied: (i) $\underline{p}_{t+1}$ must be low enough so as to bankrupt firms with T-debt, otherwise a bailout next period would not be expected and firms would not be able to take on systemic risk; (ii) $\bar{p}_{t+1}$ must be high enough so as make the risky plan preferred both to storage and a to safe plan:

$$
\pi_{t+1}^{r}\left(\underline{p}_{t+1}\right)<0, \quad Z_{t+1}^{r}>w_{t}, \quad Z_{t+1}^{r}>Z_{t+1}^{s}
$$

Next we derive equilibrium returns and determine the parameter conditions under which (58) holds. Recall that internal funds are $w_{t}=[1-\beta] p_{t} q_{t}$ if the firm is solvent or $w_{t}=\mu_{w} p_{t} q_{t}$ if it is insolvent. Using the equations for N-output and prices in (21), and noting that in an RSE the investment share $\phi_{t+1}$ equals $\phi^{l}$ if $\mathrm{N}$-firms are solvent, while $\phi_{t+1}=\phi^{c}$ if they are insolvent, it follows that equilibrium returns are

$$
\bar{R} \equiv \beta \theta \frac{\bar{p}_{t+1}}{p_{t}}=\beta \theta^{\alpha}\left[\frac{1}{\phi^{l}}\right]^{1-\alpha}, \quad \underline{R} \equiv \beta \theta \frac{p_{t+1}}{p_{t}}=\beta \theta^{\alpha}\left[\frac{1}{\phi^{l}}\right]^{1-\alpha}\left[\frac{1-\phi^{l}}{1-\phi^{c}}\right]^{1-\alpha}
$$

First, the risky plan defaults in the low price state if and only if $0>\pi^{r}\left(\underline{p}_{t+1}\right)=\beta \underline{p}_{t+1} q_{t+1}-[1+r] b_{t}=$ $\beta \underline{p}_{t+1} \frac{\theta\left[w_{t}+b_{t}\right]}{p_{t}}-\frac{1}{\delta} \frac{H}{u}\left[w_{t}+b_{t}\right]$. Using (59), this condition becomes $\pi^{r}\left(\underline{p}_{t+1}\right)=\left[\underline{R}-\frac{H}{\delta u}\right] m^{r} w_{t}<0$. Thus,

$$
\pi^{r}\left(\underline{p}_{t+1}\right)<0 \Leftrightarrow \underline{R}<\frac{1}{\delta} \frac{H}{u}
$$

Second, the risky plan is preferred to storage if and only if $[\delta u \bar{R}-H] m^{r} w_{t} \geq w_{t}$ :

$$
Z_{t+1}^{r}>w_{t} \Leftrightarrow u \delta \bar{R}-H \geq 1-\frac{H}{u} .
$$

Third, to derive $E_{t} \pi_{t+1}^{s}$, note that if an entrepreneur were to deviate and choose a safe plan, the interest rate it would have to offer is $1+\rho^{s}=[1+r] / p_{t+1}^{e}$, and her borrowing constraint would be $\delta b_{t}^{n}\left[1+\rho^{s}\right] p_{t+1}^{e} \leq H\left[w_{t}+b_{t}^{n}\right]$. Following the same steps as in the proof of Proposition 3.1, we have that $b_{t}^{n}=\left[m^{s}-1\right] w_{t}$, and the payoff would be

$$
Z_{t+1}^{s}=[u \delta \bar{R}+[1-u] \delta \underline{R}-H] m^{s} w_{t}, \quad m^{s} \equiv \frac{1}{1-H} .
$$

\footnotetext{
${ }^{52}$ To simplify notation we will omit the subscripts that indentify individual agents.
} 
Thus, a risky plan is preferred to a safe one if and only if $[u \delta \bar{R}-H] m^{r} w_{t} \geq[u \delta \bar{R}+(1-u) \delta \underline{R}-$ $H] m^{s} w_{t}$, which is equivalent to ${ }^{53}$

$$
Z_{t+1}^{r}>Z_{t+1}^{s} \Leftrightarrow u \delta \bar{R}-H \geq \delta \underline{R}[1-H / u][H / u]^{-1}
$$

Next, we verify that (60), (61) and (63) can hold simultaneously. Notice that the LHS of (61) and (63) are the same. Thus, (61) implies (63) if and only if $1-\frac{H}{u}>\delta \underline{R}\left[1-\frac{H}{u}\right](H / u)^{-1}$. Since an RSE exists only if $u>H$, we have that $1-\frac{H}{u}>0$, and so (61) implies (63) if and only if $\underline{R}<\frac{1}{\delta} \frac{H}{u}$, which is (60). Thus, (61) is stronger than (63) if and only if (60) holds. We conclude that if (60) and (61) hold, then (63) must hold.

We next determine the parameter set such that (60) and (61) hold simultaneously. Condition (61) holds if and only if

$$
\theta \geq \underline{\theta}(\delta, H, u, \alpha, \beta) \equiv\left(\left[1-\frac{H}{u}+H\right]\left[\frac{1-\beta}{1-H / u}\right]^{1-\alpha} \frac{1}{u \beta \delta}\right)^{1 / \alpha}
$$

Note that (59) implies that

$$
\underline{R} \theta^{-\alpha}=\beta\left[\left(\frac{1}{\phi^{l}}-1\right)\left(\frac{1}{1-\phi^{c}}\right)\right]^{1-\alpha}=\beta\left[\frac{\beta-H u^{-1}}{1-\beta}\right]^{1-\alpha}\left[1-\frac{\mu_{w}}{1-H}\right]^{\alpha-1}
$$

Thus, condition (60) holds if and only if

$$
\theta<\bar{\theta}\left(\delta, H, u, \alpha, \mu_{w}, \beta\right) \equiv\left[\frac{1}{\beta}\left[\frac{1-\beta}{\beta-H / u}\right]^{1-\alpha}\left[1-\frac{\mu_{w}}{1-H}\right]^{1-\alpha} \frac{H}{u \delta}\right]^{\frac{1}{\alpha}}
$$

In order for (64) and (65) to hold simultaneously it is necessary that $\bar{\theta}>\underline{\theta}$ :

$$
\begin{gathered}
\frac{1}{\beta}\left[\frac{1-\beta}{\beta-H / u}\right]^{1-\alpha}\left[1-\frac{\mu_{w}}{1-H}\right]^{1-\alpha} \frac{h}{u \delta}>\left[1-\frac{H}{u}+H\right]\left[\frac{1-\beta}{1-H / u}\right]^{1-\alpha} \frac{1}{\delta u \beta} \\
{\left[\frac{1}{\beta-H / u}\right]^{1-\alpha}\left[1-\frac{\mu_{w}}{1-H}\right]^{1-\alpha}>\left[1-\frac{H}{u}+H\right]\left[\frac{1}{1-H / u}\right]^{1-\alpha}}
\end{gathered}
$$

The LHS is decreasing in $\beta$. It ranges from infinity, for $\beta \rightarrow \underline{\beta} \equiv H / u$, to $L(1)=\left[\frac{1}{1-H / u}\right]^{1-\alpha}\left[1-\frac{\mu_{w}}{1-H}\right]^{1-\alpha}$ for $\beta=1$. Since $L(1)$ is lower than the RHS (because $u>H$ ), it follows that there is a unique threshold $\bar{\beta}$ such that $\bar{\theta}>\underline{\theta}$ if and only if $\beta<\bar{\beta}$. The above condition implies that this upper bound on $\beta$ is

$$
\bar{\beta}\left(H, u, \alpha, \mu_{w}\right)=\underline{\beta}+\left[1-\frac{\mu_{w}}{1-H}\right]\left[\frac{1}{H}-\frac{1}{u}+1\right]^{\frac{-1}{1-\alpha}}\left[\frac{1}{1-H / u}\right]^{-1}
$$

\footnotetext{
${ }^{53}$ Rewrite $[\delta u \bar{R}-H] m^{r} w_{t} \geq[\delta u \bar{R}+\delta(1-u) \underline{R}-H] m^{s} w_{t}$ as $[\delta u \bar{R}-H]\left[m^{r}-m^{s}\right] \geq \delta(1-u) \underline{R} m^{s} \Leftrightarrow[\delta u \bar{R}-$ $H]\left[\frac{1-u}{u} H m^{r} m^{s}\right] \geq \delta(1-u) \underline{R} m^{s} \Leftrightarrow[\delta u \bar{R}-H]\left[m^{r}-1\right] \geq \delta \underline{R}$.
} 
Summing up, given that parameters $(\delta, H, u, \beta)$ satisfy (11), condition (60) holds if and only if $\theta<\bar{\theta}$, while condition (61) holds if and only if $\theta>\underline{\theta}$. Furthermore, $\bar{\theta}>\underline{\theta}$ if and only if $\beta<\bar{\beta}$. Thus, we conclude that during a no-crisis period, equilibrium expected returns are such that an entrepreneur prefers the equilibrium risky plan over both storage and a safe plan (i.e., the conditions in (59) hold) if and only if $\theta \in(\underline{\theta}, \bar{\theta})$ and $\beta<\bar{\beta}$.

Consider next a crisis period. Given that all other entrepreneurs choose a safe plan, there can be no crisis and no bailout in the post-crisis period. Thus, an entrepreneur faces the same problem as that in a safe symmetric equilibrium. It follows from Proposition 3.1 that she will find it optimal to choose a safe investment plan if and only if $\beta \theta p_{t+1} / p_{t} \geq \delta^{-1}$. This condition is equivalent to $\beta \theta^{\alpha}\left(\phi^{s}\right)^{\alpha-1} \geq \delta^{-1}$, which is implied by (61) because $u>H$ and $\left(\phi^{l}\right)^{\alpha-1}<\left(\phi^{s}\right)^{\alpha-1}$.

Finally, notice that lenders do not fund any diversion plan because they will get repaid zero.

Part B. We show that two crises cannot occur in consecutive periods. Suppose to the contrary that if a crisis occurs at $\tau$, firms choose risky plans at $\tau$. We will show that it is not possible for firms to become insolvent in the low price state at $\tau+1$ (i.e., $\pi\left(\underline{p}_{\tau+1}\right)<0$ ), and so a bailout cannot be expected. It suffices to consider the case in which firms internal funds at $\tau+1$ equal $\mu_{w}$, and they undertake safe plans at $\tau+1$, as this generates the lowest possible price $\underline{p}_{\tau+1}$. We will show that even in these extreme case it is not possible to generate $\pi\left(\underline{p}_{\tau+1}\right)<0$. Along this path the N-investment share is $\phi_{\tau}=\tilde{\phi}^{c}:=\mu_{w} m^{r}$ and $\phi_{\tau+1}=\phi^{c}:=\mu_{w} m^{s}$. Thus,

$$
\tilde{\pi}\left(\underline{p}_{\tau+1}\right)=\beta \underline{p}_{\tau+1} q_{\tau+1}-L_{\tau+1}=\left[\frac{\beta \theta \underline{p}_{\tau+1}}{p_{\tau}}-\frac{H}{\delta u}\right] m^{r} \mu_{w}=\left[\beta \theta \frac{\alpha\left[1-\phi^{c}\right]^{\alpha-1}\left[\theta \tilde{\phi}^{c} q_{\tau}\right]^{\alpha-1}}{\alpha\left[1-\tilde{\phi}^{c}\right]^{\alpha-1}\left[q_{\tau}\right]^{\alpha-1}}-\frac{H}{\delta u}\right] \tilde{\phi}^{c}
$$

In order to get $\tilde{\pi}\left(\underline{p}_{\tau+1}\right)<0$ it is necessary that

$$
\delta u \beta \theta^{\alpha} \frac{\left[1-\phi^{c}\right]^{\alpha-1}\left[\tilde{\phi}^{c}\right]^{\alpha-1}}{\left[1-\tilde{\phi}^{c}\right]^{\alpha-1}}<H \quad \Leftrightarrow \quad \delta u \beta \theta^{\alpha}\left[\frac{1}{\tilde{\phi}^{c}}\right]^{1-\alpha}<H\left[\frac{1-\phi^{c}}{1-\tilde{\phi}^{c}}\right]^{1-\alpha}
$$

Recall that a RSE requires that a risky plan be preferred to storage, i.e., condition (61): $\delta u \beta \theta^{\alpha}\left[\frac{1}{\phi^{l}}\right]^{1-\alpha} \geq$ $H+1-\frac{H}{u}$. Since $1-\frac{H}{u}>0$ (because a necessary condition for an RSE is $u>H$ ), condition (61) implies $\delta u \beta \theta^{\alpha}\left[\frac{1}{\phi^{l}}\right]^{1-\alpha} \geq H$. Notice also that $\phi^{l} \equiv[1-\beta] m^{r}>\mu_{w} m^{r} \equiv \tilde{\phi}^{c}$ because we have imposed financial distress costs of crisis: $w_{\text {crisis }}=\mu_{w}<1-\beta$. Combining these facts we have

$$
\delta u \beta \theta^{\alpha}\left[\frac{1}{\tilde{\phi}^{c}}\right]^{1-\alpha}>\delta u \beta \theta^{\alpha}\left[\frac{1}{\phi^{l}}\right]^{1-\alpha}>H
$$

Finally, notice that $(68)$ contradicts $(67)$ because $\left[\frac{1-\phi^{c}}{1-\tilde{\phi}^{c}}\right]>1$. Hence, in an RSE it is not possible for agents to choose a risky plan during a crisis period. 
Proof of Lemma 3.1. Propositions 3.1 and 3.2 state that (i) if an RSE exists, then parameters satisfy (11) and the input sector productivity $\theta>\underline{\theta}$; while (ii) an SSE exists if and only if (11) holds and $\theta>\underline{\theta}^{s}$. Therefore, to prove Lemma 3.1 it suffices to show $\underline{\theta}>\underline{\theta}^{s}$ for all parameters satisfying (11): $H<1, u \in(H, 1)$ and $\beta>H / u$. To verify this is the case rewrite the lower bounds as follows:

$$
\underline{\theta}^{s}=\left(\frac{1}{\delta \beta}\right)^{\frac{1}{\alpha}}\left(\phi^{s}\right)^{\frac{1-\alpha}{\alpha}}, \quad \underline{\theta}=(A(u))^{\frac{1}{\alpha}}\left(\frac{1}{\delta \beta}\right)^{\frac{1}{\alpha}}\left(\phi^{l}\right)^{\frac{1-\alpha}{\alpha}}, A(u) \equiv\left(1-\frac{H}{u}+H\right) \frac{1}{u} .
$$

Since $\alpha \in(0,1)$ in the T-good production function $(3)$, we have that $\left(\phi^{l}\right)^{\frac{1-\alpha}{\alpha}}>\left(\phi^{s}\right)^{\frac{1-\alpha}{\alpha}}$ for any $u \in(H, 1)$. Thus, it suffices to show that $A(u) \geq 1$ for all $u \in(H, 1)$. Using the change of variable $x=1 / u$, this condition can be rewritten as $A(x) \geq 1$ for all $x \in(1,1 / H)$. This condition holds because: (i) $A(x)=(1+H) x-H x^{2}$ is a concave parabola, so $A(x)$ is greater than $A(x=1)$ and $A\left(x=\frac{1}{H}\right)$ for any $x \in(1,1 / H)$; (ii) since $A(x=1)=1$ and $A\left(x=\frac{1}{H}\right)=1$, we have that $A(x)>1$ for all $x \in(1,1 / H)$. Equivalently, $A(u)>1$ for all $u \in(H, 1)$. Hence, $\underline{\theta}^{s}<\underline{\theta}$ if (11) holds.

Proof of Lemma 3.2. We have shown in the text that $\phi^{s}<\phi^{c p}$. Here we show that $\phi^{l}<\phi^{c p}$. This condition is equivalent to $\theta>\left(\frac{1}{\delta}\right)^{\frac{1}{\alpha}}\left(\phi^{l}\right)^{\frac{1-\alpha}{\alpha}} \equiv \theta^{\prime \prime}$. Since an RSE exists only if $\theta>\underline{\theta} \equiv$ $\left(\left[1-\frac{H}{u}+H\right] \frac{1}{\delta u \beta}\right)^{\frac{1}{\alpha}}\left(\phi^{l}\right)^{\frac{1-\alpha}{\alpha}}$, it follows that $\phi^{l}<\phi^{c p}$ if the lower bound $\theta^{\prime \prime}$ is smaller than $\underline{\theta}$. This condition is equivalent to $\left(\frac{1}{\delta}\right)^{\frac{1}{\alpha}}<\left(\left[1-\frac{H}{u}+H\right] \frac{1}{\delta u \beta}\right)^{\frac{1}{\alpha}}$. Thus,

$$
\phi^{l}<\phi^{c p} \text { if } \frac{1}{\delta}<\left[1-\frac{H}{u}+H\right] \frac{1}{\delta u \beta} \Longleftrightarrow u \beta<\left[1-\frac{H}{u}+H\right] .
$$

Rewriting $1-\frac{H}{u}+H$ as $1-\frac{H}{u}[1-u]$ and using the restriction $\frac{H}{u}<1$ in (11), we have that $1-\frac{H}{u}+H=1-\frac{H}{u}[1-u]>1-[1-u]=u$. Since $\beta<1$, it follows that $u \beta<1-\frac{H}{u}+H$. Hence, $\phi^{l}<\phi^{c p}$ if necessary condition (11) for an RSE holds. $\square$

Proof of Proposition 3.3. Here, we derive the limit distribution of GDP's compounded growth rate $\left(\log \left(g d p_{t}\right)-\log \left(g d p_{t-1}\right)\right)$ along the RSE characterized in Proposition 3.2. In this RSE, firms choose safe plans in a crisis period and resume risk-taking the period immediately after the crisis. It follows from (20), (27) and (28) that the growth process follows a three-state Markov chain characterized by

$$
\Gamma=\left(\begin{array}{l}
\log \left(\left(\theta \phi^{l}\right)^{\alpha}\right) \\
\log \left(\left(\theta \phi^{l}\right)^{\alpha} \frac{Z\left(\phi^{c}\right)}{Z\left(\phi^{l}\right)}\right) \\
\log \left(\left(\theta \phi^{c}\right)^{\alpha} \frac{Z\left(\phi^{l}\right)}{Z\left(\phi^{c}\right)}\right)
\end{array}\right), \quad T=\left(\begin{array}{ccc}
u & 1-u & 0 \\
0 & 0 & 1 \\
u & 1-u & 0
\end{array}\right)
$$

The three elements of $\Gamma$ are the growth rates in the lucky, crisis and post-crisis states, respectively. The element $T_{i j}$ of the transition matrix is the transition probability from state $i$ to state $j$. Since 
the transition matrix is irreducible, the growth process converges to a unique limit distribution over the three states that solves $T^{\prime} \Pi=\Pi$. Thus, $\Pi=\left(\frac{u}{2-u}, \frac{1-u}{2-u}, \frac{1-u}{2-u}\right)^{\prime}$, where the elements of $\Pi$ are the shares of time that an economy spends in each state over the long-run. It then follows that the mean long-run GDP growth rate is $E\left(1+\gamma^{r}\right)=\exp \left(\Pi^{\prime} \Gamma\right)$, that is:

$$
E\left(1+\gamma^{r}\right)=\left(1+\gamma^{l}\right)^{\omega}\left(1+\gamma^{c r}\right)^{1-\omega}=\theta^{\alpha}\left(\phi^{l}\right)^{\alpha \omega}\left(\phi^{l} \phi^{c}\right)^{\alpha \frac{1-\omega}{2}}, \quad \text { where } \omega=\frac{u}{2-u},
$$

which can be expressed as:

$$
E\left(1+\gamma^{r}\right)=\left(\theta \phi^{s}\right)^{\alpha}\left(\frac{\phi^{l}}{\phi^{s}}\right)^{\frac{1}{2-u}}\left(\frac{\mu_{w}}{1-\beta}\right)^{\frac{1-u}{2-u}}
$$

Since growth in a safe equilibrium is $1+\gamma^{s}=\left(\theta \phi^{s}\right)^{\alpha}$, we have

$$
\frac{E\left(1+\gamma^{r}\right)}{1+\gamma^{s}}=\left(\frac{\phi^{l}}{\phi^{s}}\right)^{\frac{1}{2-u}}\left(\frac{\mu_{w}}{1-\beta}\right)^{\frac{1-u}{2-u}}=\left(\frac{1-H}{1-H u^{-1}}\right)^{\frac{1}{2-u}}\left(\frac{\mu_{w}}{1-\beta}\right)^{\frac{1-u}{2-u}}
$$

It follows that for any crisis probability for which an RSE exists (i.e., $u \in(H, 1))$ :

$$
\begin{aligned}
E\left(\gamma^{r}\right) & >\gamma^{s} \Leftrightarrow \log \left(\phi^{l}\right)-\log \left(\phi^{s}\right)>[1-u]\left[\log (1-\beta)-\log \left(\mu_{w}\right)\right] \\
& \Leftrightarrow \frac{\mu_{w}}{1-\beta}>\left(\frac{1-H u^{-1}}{1-H}\right)^{\frac{1}{1-u}} .
\end{aligned}
$$

If $u=1$, both growth rates are the same, as we can see in (71). Thus, to determine whether $E\left(\gamma^{r}\right)$ is greater or smaller than $\gamma^{s}$ for $u \in(H, 1)$, we analyze the relation between $\frac{E\left(1+\gamma^{r}\right)}{1+\gamma^{s}}$ and $u$.

$$
\begin{aligned}
F(u) & \equiv \frac{\partial \log \left(E\left(1+\gamma^{r}\right) /\left(1+\gamma^{s}\right)\right)}{\partial u} \\
& =\frac{1}{(2-u)^{2}} \log \left(\frac{1-H}{1-H u^{-1}}\right)-\frac{1}{2-u} \frac{H(1-H)}{u^{2}\left(1-H u^{-1}\right)^{2}}\left(\frac{1-H}{1-H u^{-1}}\right)^{-1}-\frac{1}{(2-u)^{2}} \log \left(\frac{\mu_{w}}{1-\beta}\right) \\
& =\frac{1}{(2-u)^{2}} \log \left(\frac{1-H}{1-H u^{-1}}\right)-\frac{1}{2-u} \frac{H(1-H)}{u^{2}\left(1-H u^{-1}\right)^{2}} \frac{1-H u^{-1}}{1-H}-\frac{1}{(2-u)^{2}} \log \left(\frac{\mu_{w}}{1-\beta}\right) \\
& =\frac{1}{(2-u)^{2}} \log \left(\frac{1-H}{1-H u^{-1}}\right)-\frac{1}{2-u} \frac{H}{u^{2}\left(1-H u^{-1}\right)}-\frac{1}{(2-u)^{2}} \log \left(\frac{\mu_{w}}{1-\beta}\right) \\
& =\frac{1}{(2-u)^{2}}\left[\log \left(\frac{1-H}{1-H u^{-1}}\right)-\frac{2-u}{u^{2}} \frac{H}{1-H u^{-1}}-\log \left(\frac{\mu_{w}}{1-\beta}\right)\right] \\
& =\frac{1}{(2-u)^{2}}\left[\log \left(\frac{1-H}{1-H u^{-1}} \cdot \frac{1-\beta}{\mu_{w}}\right)-\left(\frac{2-u}{u^{2}} \cdot \frac{H}{1-H u^{-1}}\right)\right] .
\end{aligned}
$$

It follows from (73) that $F(u)$ is decreasing in $u$ if and only if

$$
\frac{\partial \log \left(E\left(\gamma^{r}\right) / \gamma^{s}\right)}{\partial u}<0 \Leftrightarrow \frac{\mu_{w}}{1-\beta}>\frac{1-H}{1-H u^{-1}} \exp \left(-\frac{2-u}{u^{2}} \frac{H}{1-H u^{-1}}\right)
$$


Next, we determine the values of $u \in(H, 1)$ for which $(74)$ holds. First, we show that condition (74) becomes less stringent as $u$ decreases, i.e., the RHS of $(74)$ is increasing in $u$ over $(H, 1)$. Then we compute $\lim _{u \uparrow 1} F(u)$ and $\lim _{u \downarrow H} F(u)$.

$$
\begin{aligned}
\frac{\partial \log (R H S(74))}{\partial u} & =\frac{1}{u^{2}}\left[\frac{-H}{1-H u^{-1}}+\left(\frac{2}{u^{2}}-\frac{1}{u}\right) \frac{(H)^{2}}{\left(1-H u^{-1}\right)^{2}}+\left(\frac{4}{u}-1\right) \frac{H}{1-H u^{-1}}\right] \\
& =\frac{1}{u^{2}}\left[2\left(\frac{2}{u}-1\right) \frac{H}{1-H u^{-1}}+\frac{1}{u}\left(\frac{2}{u}-1\right)\left(\frac{H}{1-H u^{-1}}\right)^{2}\right]
\end{aligned}
$$

This expression is unambiguously positive for all $u \in(H, 1)$.

Equation (73) implies that $\lim _{u \uparrow 1} F(u)=\left[\log \left(\frac{1-\beta}{\mu_{w}}\right)-\frac{H}{1-H}\right]$. Thus,

$$
\lim _{u \uparrow 1} F(u)<0 \Leftrightarrow-\frac{H}{1-H}<\log \left(\frac{\mu_{w}}{1-\beta}\right) \Leftrightarrow e^{-\frac{H}{1-H}}<\frac{\mu_{w}}{1-\beta} .
$$

Using again (73), we have that

$$
\begin{aligned}
& \lim _{u \downarrow H} F(u)=\lim _{u \downarrow H}\left\{\frac{1}{(2-H)^{2}} \log \left(\frac{1-H}{1-H u^{-1}}\right)-\frac{1}{2-H} \frac{1}{H\left(1-H u^{-1}\right)}-\left(\frac{1}{2-H}\right)^{2} \log \left(\frac{\mu_{w}}{1-\beta}\right)\right\} \\
& =\lim _{u \downarrow H}\left[\frac{1}{2-H} \log \left(\frac{1}{1-H u^{-1}}\right)-\frac{1}{H} \frac{1}{1-H u^{-1}}\right] \frac{1}{2-H}+\frac{\log (1-H)}{(2-H)^{2}}-\left(\frac{1}{2-H}\right)^{2} \log \left(\frac{\mu_{w}}{1-\beta}\right)
\end{aligned}
$$

The bracketed term has the form $a \ln (x)-b x$ with $x=\frac{1}{1-H u^{-1}}, \quad a=\frac{1}{2-H}$ and $b=\frac{1}{H}$. Since $\lim _{x \rightarrow \infty}(a \ln (x)-b x)=-\operatorname{sgn}(b) \cdot \infty$, we have that $\lim _{u \downarrow H}\left[\frac{1}{2-H} \log \left(\frac{1}{1-H u^{-1}}\right)-\frac{1}{H} \frac{1}{1-H u^{-1}}\right]=$ $-\operatorname{sgn}\left(\frac{1}{H}\right) \cdot \infty$. Hence, $\lim _{u \downarrow H} F(u)<0$ for all $\mu_{w} \in(0,1-\beta)$.

Summing up, we have shown that: (i) If $u \downarrow H$, condition (74) holds for any $\mu_{w} \in(0,1-\beta)$, while if $u \uparrow 1,(74)$ holds only if and only if $\frac{\mu_{w}}{1-\beta}>e^{-\frac{H}{1-H}}$; (ii) condition (74) becomes less stringent as $u$ falls. Thus, there are 2 cases depending on the size of $\frac{\mu_{w}}{1-\beta}$ :

$$
\begin{aligned}
& \text { If } \frac{\mu_{w}}{1-\beta}>e^{-\frac{H}{1-H}}, \quad \text { then } \frac{\partial \log \left(E\left(\gamma^{r}\right) / \gamma^{s}\right)}{\partial u}<0 \text { for all } u \in(H, 1) \\
& \text { If } \frac{\mu_{w}}{1-\beta}<e^{-\frac{H}{1-H}}, \text { then } \frac{\partial \log \left(E\left(\gamma^{r}\right) / \gamma^{s}\right)}{\partial u}\left\{\begin{array}{c}
<0 \text { for } u \in\left(H, u^{*}\right) \\
>0 \text { for } u \in\left(u^{*}, 1\right)
\end{array}\right.
\end{aligned}
$$

We know from (71) that if $u=1$, then $E\left(1+\gamma^{r}\right)=\left(1+\gamma^{s}\right)$. Thus, it follows from (76) that $\frac{\mu_{w}}{1-\beta}>e^{-\frac{H}{1-H}}$ is a sufficient condition for $E\left(1+\gamma^{r}\right)>\left(1+\gamma^{s}\right)$ for all $u \in(H, 1)$. Part 1 of Proposition 3.3 follows directly by expressing this conditions in term of crisis financial distress costs $l^{d} \equiv 1-\frac{\mu_{w}}{1-\beta}$. That is, $l^{d}<1-e^{-\frac{H}{1-H}}$. A more general result than Proposition 3.3 would add that "if $l^{d} \geq 1-e^{-\frac{H}{1-H}}$, there exists a threshold $u^{*}>H$, such that $E\left(1+\gamma^{r}\right)>\left(1+\gamma^{s}\right)$ for all $u \in\left(H, u^{*}\right)$. However, $E\left(1+\gamma^{r}\right)<\left(1+\gamma^{s}\right)$ for $u \in\left(u^{*}, 1\right)$." That is, when financial distress costs are large, liberalization increases mean growth only if the crisis probability is high enough, within the admissible parameter region. 
Part 2 follows directly from Lemma 3.2. Part 3 follows from the fact that condition (74) becomes less stringent as $u$ falls (i.e., the RHS of (74) is strictly increasing in $u$ ). To get some intuition rewrite (73) as follows:

$$
\frac{\partial \log \left(E\left(1+\gamma^{r}\right) /\left(1+\gamma^{s}\right)\right)}{\partial u}=\frac{1}{(2-u)^{2}}[\underbrace{\log \left(\frac{\phi^{l}}{\phi^{c}}\right)}_{\text {COSTS }}-\underbrace{\left(\frac{2-u}{u} \cdot \frac{b_{t}^{\text {risky }}}{w_{t}}\right)}_{\text {BENEFITS }}]
$$

In the last equality we have used the risky equilibrium borrowing constraint $b_{t}^{r i s k y}=\frac{H u^{-1}}{1-H u^{-1}} w_{t}$. $\square$

The Length of Recovery. The lenght of the recovery phase is the minimum period it takes for an economy to reach back its pre-crisis level starting from two years after the onset of the crisis. Assuming that the economy follows a safe path until the pre-crisis level output is reached, the cumulative growth rate from the onset of the crisis to the end of the recovery is given by:

$$
F(T)=\left(\theta \phi^{l}\right)^{\alpha}\left(\theta \phi^{c}\right)^{\alpha} \frac{Z\left(\phi^{s}\right)}{Z\left(\phi^{l}\right)}\left(\theta \phi_{s}\right)^{\alpha(T)} .
$$

Therefore $T$ is implicitely defined by $F(T)=1$ :

$$
T=-\frac{\left(\ln \theta \phi^{l}+\ln \theta \phi^{c}\right)+\frac{1}{\alpha}\left(\operatorname { l n } \left(Z\left(\phi^{s}\right)-\ln \left(Z\left(\phi^{l}\right)\right)\right.\right.}{\ln \left(\theta \phi_{s}\right)}
$$

In the absence of a cool-off period, the length of the recovery phase is computed as $T^{*}=-\frac{\ln \theta \phi^{c}}{\ln \theta \phi^{l}}$.

Proof of Proposition 5.1. We prove that bailout costs can be financed via domestic taxation (i.e., that (7) holds) by showing that $W^{r}$ (defined in (38)) is positive. To simplify notation we assume, temporarily, that there is only one crisis (at time $\tau$ ). It follows that the bailout cost are

$$
T(\tau)=L_{\tau-1}=\frac{\alpha}{1-\phi^{l}} \frac{H}{u \delta} \phi^{l} y_{\tau-1} .
$$

To derive (77) notice that the borrowing constraint implies $L_{\tau-1} \equiv \frac{1}{\delta} b_{\tau-1}=\frac{1}{\delta} \frac{H}{u}\left[w_{\tau-1}+b_{\tau-1}\right]$. Since the firm's budget constraint implies $w_{\tau-1}+b_{\tau-1}=\phi^{l} p_{\tau-1} q_{\tau-1}$, the market clearing condition is $p_{\tau-1} q_{\tau-1}\left[1-\phi_{\tau-1}\right]=\alpha y_{\tau-1}$, and $\phi_{\tau-1}=\phi^{l}$, we have that $w_{\tau-1}+b_{\tau-1}=\phi^{l} \frac{\alpha}{1-\phi^{l}} y_{\tau-1}$. It follows that profits are:

$$
\begin{aligned}
& \pi_{t}=\frac{\alpha}{1-\phi^{l}} \beta y_{t}-\frac{\alpha \phi^{l}}{1-\phi^{l}} \frac{H}{u \delta} y_{t-1}, \quad t \neq\{0, \tau, \tau+1\} \\
& \pi_{0}=\frac{\alpha}{1-\phi^{l}} \beta y_{0}, \quad \pi_{\tau}=0, \quad \pi_{\tau+1}=\frac{\alpha}{1-\phi^{l}} \beta y_{\tau+1}-\frac{\alpha \phi^{c}}{1-\phi^{c}} \frac{H}{\delta} y_{\tau}
\end{aligned}
$$


Replacing these expressions in (38) and using market clearing condition $p_{t} q_{t}\left[1-\phi_{t}\right]=\alpha y_{t}$, we get

$$
\begin{aligned}
W^{r}(\tau)= & (1-\alpha) y_{o}+\frac{\alpha \beta y_{o}}{1-\phi^{l}}+\sum_{t=1}^{\tau-1} \delta^{t}\left[\left[(1-\alpha) y_{t}+\frac{\alpha \beta y_{t}}{1-\phi^{l}}-\frac{\alpha \phi^{l} y_{t-1}}{1-\phi^{l}} \frac{H}{u \delta}\right]\right. \\
& +\delta^{\tau}\left[(1-\alpha) y_{\tau}-\frac{\alpha \phi^{l} y_{\tau-1}}{1-\phi^{l}} \frac{H}{u \delta}\right] \\
& +\delta^{\tau+1}\left[(1-\alpha) y_{\tau+1}+\frac{\alpha}{1-\phi^{l}} \beta y_{\tau+1}-\frac{H}{\delta} \frac{\alpha \phi^{c}}{1-\phi^{c}} y_{\tau}\right] \\
& +\sum_{t=\tau+2}^{\infty} \delta^{t}\left[(1-\alpha) y_{t}+\frac{\alpha \beta}{1-\phi^{l}} y_{t}-\frac{\alpha \phi^{l}}{1-\phi^{l}} \frac{H}{u \delta} y_{t-1}\right] \\
= & \sum_{t \neq \tau} \delta^{t}\left[(1-\alpha) y_{t}+\frac{\alpha}{1-\phi^{l}} \beta y_{t}-\delta \frac{\alpha}{1-\phi^{l}} \frac{H}{u \delta} \phi^{l} y_{t}\right] \\
& +\delta^{\tau}\left[(1-\alpha) y_{\tau}-\delta \frac{\alpha \phi^{c}}{1-\phi^{c}} \frac{H}{u \delta} y_{\tau}\right] \\
= & \sum_{t \neq \tau} \delta^{t}\left[(1-\alpha) y_{t}+\frac{\alpha}{1-\phi^{l}} \beta y_{t}-\frac{\alpha}{1-\phi^{l}} \frac{H}{u} \phi^{l} y_{t}\right] \\
& +\delta^{\tau}\left[(1-\alpha) y_{\tau}-\frac{\alpha \phi^{c}}{1-\phi^{c}} H y_{\tau}\right] \\
= & \sum_{t \neq \tau} \delta^{t}\left[(1-\alpha) y_{t}+\frac{\alpha y_{t}}{1-\phi^{l}}\left(\beta-\frac{H}{u} \phi^{l}\right)\right]+\delta^{\tau}\left[(1-\alpha) y_{\tau}-\frac{\alpha \phi^{c}}{1-\phi^{c}} H y_{\tau}\right] \\
= & \sum_{t \neq \tau} \delta^{t} y_{t}+\kappa^{c} y_{\tau}, \quad \frac{\alpha}{1-\phi^{c}} H \phi^{c}=1-\frac{\alpha\left[1-\mu_{w}\right]}{1-\phi^{c}} .
\end{aligned}
$$

Simplifying further we get

$$
\begin{aligned}
W^{r}(\tau) & =\sum_{t \neq \tau} \delta^{t}\left[(1-\alpha) y_{t}+\frac{\alpha y_{t}}{1-\phi^{l}}\left(\beta-\frac{H}{u} \frac{1-\beta}{1-\frac{H}{u}}\right)\right]+\delta^{\tau}\left[(1-\alpha) y_{\tau}-\frac{\alpha \phi^{c}}{1-\phi^{c}} H y_{\tau}\right] \\
& =\sum_{t \neq \tau} \delta^{t}\left[(1-\alpha) y_{t}+\frac{\alpha y_{t}}{1-\phi^{l}} \frac{1}{1-\frac{H}{u}}\left(\beta-\frac{H}{u}\right)\right]+\delta^{\tau}\left[(1-\alpha) y_{\tau}-\frac{\alpha \phi^{c}}{1-\phi^{c}} H y_{\tau}\right] \\
& =\sum_{t \neq \tau} \delta^{t}\left[(1-\alpha) y_{t}+\frac{\alpha y_{t}}{\frac{\left(\beta-\frac{H}{u}\right)}{1-\frac{H}{u}}} \frac{1}{1-\frac{H}{u}}\left(\beta-\frac{H}{u}\right)\right]+\delta^{\tau}\left[(1-\alpha) y_{\tau}-\frac{\alpha \phi^{c}}{1-\phi^{c}} H y_{\tau}\right] \\
& =\sum_{t \neq \tau} \delta^{t}\left[(1-\alpha) y_{t}+\alpha y_{t}\right]+\delta^{\tau}\left[(1-\alpha) y_{\tau}-\frac{\alpha \phi^{c}}{1-\phi^{c}} H y_{\tau}\right] \\
& =\sum_{t \neq \tau} \delta^{t} y_{t}+\delta^{\tau} \kappa^{c} y_{\tau}, \quad \kappa^{c} \equiv 1-\alpha-\frac{\alpha}{1-\phi^{c}} H \phi^{c} .
\end{aligned}
$$

We can simplify $\kappa^{c}$ by using $\phi^{c}=\frac{\mu_{w}}{1-H}$ :

$$
\begin{aligned}
\kappa^{c} & =1-\alpha\left(1+\frac{1}{1-\phi^{c}} H \phi^{c}\right)=1-\frac{\alpha}{1-\phi^{c}}\left(1+\phi^{c}(H-1)\right) \\
& =1-\frac{\alpha}{1-\phi^{c}}\left(1+\frac{\mu_{w}}{1-H}(H-1)\right)=1-\frac{\alpha\left[1-\mu_{w}\right]}{1-\phi^{c}}
\end{aligned}
$$


If we allow for the possibility of multiple crises, then

$$
W^{r}=E_{0} \sum_{t=0}^{\infty} \delta^{t} \kappa_{t} y_{t}, \quad \kappa_{t}= \begin{cases}\kappa^{c} \equiv 1-\frac{\alpha\left[1-\mu_{w}\right]}{1-\phi^{c}} & \text { if } t=\tau_{i} \\ 1 & \text { otherwise }\end{cases}
$$

where, $\tau_{i}$ denotes a time of crisis. In order to compute this expectation, we need to calculate the limit distribution of $\kappa_{t} y_{t} \equiv \tilde{y}_{t}$, which corresponds to T-output net of bankruptcy costs. To derive this limit distribution notice that $\frac{\tilde{y}_{t}}{\tilde{y}_{t-1}}$ follows a three-state Markov chain defined by:

$$
\widetilde{T}=\left(\begin{array}{ccc}
u & 1-u & 0 \\
0 & 0 & 1 \\
u & 1-u & 0
\end{array}\right), \quad \widetilde{G}=\left(\begin{array}{c}
g_{1} \\
g_{2} \\
g_{3}
\end{array}\right)=\left(\begin{array}{c}
\left(\theta \phi^{l}\right)^{\alpha} \\
{\left[\theta \phi^{l} \frac{1-\phi^{c}}{1-\phi^{l}}\right]^{\alpha} \kappa_{c}} \\
{\left[\theta \phi^{c} \frac{1-\phi^{l}}{1-\phi^{c}}\right]^{\alpha} \frac{1}{\kappa_{c}}}
\end{array}\right)
$$

To derive $W^{r}$ in closed form consider the following recursion

$$
\begin{aligned}
V\left(\widetilde{y}_{0}, g_{0}\right) & =E_{0} \sum_{t=0}^{\infty} \delta^{t} \widetilde{y}_{t}=\widetilde{y}_{0}+\delta E_{0} V\left(\widetilde{y}_{1}, g_{1}\right) \\
V\left(\widetilde{y}_{t}, g_{t}\right) & =y_{t}+\beta E_{t} V\left(\widetilde{y}_{t+1}, g_{t+1}\right)
\end{aligned}
$$

Suppose that the function $V$ is linear: $V\left(\widetilde{y}_{t}, g_{t}\right)=\widetilde{y}_{t} w\left(g_{t}\right)$, with $w\left(g_{t}\right)$ an undetermined coefficient. Substituting this guess into (81), we get $w\left(g_{t}\right)=1+\delta E_{t} g_{t+1} w\left(g_{t+1}\right)$. Combining this condition with (80), it follows that $w\left(g_{t+1}\right)$ satisfies

$$
\left(\begin{array}{l}
w_{1} \\
w_{2} \\
w_{3}
\end{array}\right)=\left(\begin{array}{l}
1 \\
1 \\
1
\end{array}\right)+\delta\left(\begin{array}{ccc}
u & 1-u & 0 \\
0 & 0 & 1 \\
u & 1-u & 0
\end{array}\right)\left(\begin{array}{l}
g_{1} w_{1} \\
g_{2} w_{2} \\
g_{3} w_{3}
\end{array}\right) \Rightarrow \begin{aligned}
& w_{1}=\frac{1+(1-u) \delta g_{2}}{1-(1-u) \delta^{2} g_{2} g_{3}-u \delta g_{1}} \\
& w_{2}=\frac{1+\delta g_{3}-u \delta g_{1}}{1-(1-u) \delta^{2} g_{2} g_{3}-u \delta g_{1}} \\
& w_{3}=\frac{1+(1-u) \delta g_{2}}{1-(1-u) \delta^{2} g_{2} g_{3}-u \delta g_{1}}
\end{aligned}
$$

This solution exists and is unique if and only if $1>g_{1} \delta u+g_{2} g_{3} \delta^{2}(1-u)$, or equivalently

$$
\left[\theta \phi^{l}\right]^{\alpha} \delta u+\left[\theta^{2} \phi^{l} \phi^{c}\right]^{\alpha} \delta^{2}(1-u)<1
$$

We show that $(82)$ holds if the central planner's problem has a interior solution $\left(\phi^{c p}<1\right)$. Recall that $\phi^{c p}=\left(\theta^{\alpha} \delta\right)^{\frac{1}{1-\alpha}}<1 \Leftrightarrow \theta^{\alpha} \delta<1$, and that $\phi^{s}<\phi^{l}<\phi^{c p}$ by Lemma 3.2. Thus,

$$
\begin{aligned}
{\left[\theta \phi^{c}\right]^{\alpha} } & <\left[\theta \phi^{l}\right]^{\alpha}<\left[\theta \phi^{c p}\right]^{\alpha}<1 \\
& \Longrightarrow\left[\theta \phi^{l}\right]^{\alpha} \delta u+\left[\theta^{2} \phi^{l} \phi^{c}\right]^{\alpha} \delta^{2}(1-u)<\delta(1-u)+\delta^{2} u<1 .
\end{aligned}
$$

Since at time 0 the economy is in the tranquil state (i.e., $\left.V\left(y_{0}, g_{0}\right)=w_{1} y_{0}^{l}\right)$ and since $g_{2} g_{3}=$ $\left(\theta \phi^{l}\right)^{\alpha}\left(\theta \phi^{c}\right)^{\alpha}$, it follows that $W^{r}$ is given by (41). Notice that if $\phi^{c p}<1$, the denominator of $W^{r}$ 
is unabiguously positive because (82) holds. Thus, to establish that $W^{r}>0$ we just need to show that (41)'s numerator is positive. Rewrite the numerator of (41) as follows:

$$
\begin{aligned}
N & \equiv\left(1-\phi^{l}\right)^{\alpha}+\delta \theta^{\alpha}(1-u)\left(\phi^{l}\right)^{\alpha}\left(1-\phi^{c}\right)^{\alpha} \kappa^{c}, \quad \text { where } \\
\kappa^{c} & \equiv 1-\frac{\alpha\left[1-\mu_{w}\right]}{1-\phi^{c}}=1-\alpha-\frac{\mu_{w} \alpha H}{1-H-\mu_{w}}
\end{aligned}
$$

We will show that $N>0$ by considering a sequence of lower bounds on (83), and showing that they are positive for all admissible parameter values. Notice that $N$ is decreasing in $\mu_{w}$ because $\mu_{w}<1-\beta<1-H, H<1$, and $\alpha<1$. Thus, we obtain a lower bound for $N$ by setting $\mu_{w}=1-\beta$.

$$
N>N_{1} \equiv\left(1-\phi^{l}\right)^{\alpha}+\delta \theta^{\alpha}(1-u)\left(\phi^{l}\right)^{\alpha}\left(1-\frac{1-\beta}{1-H}\right)^{\alpha}\left(1-\frac{\alpha \beta}{1-\frac{1-\beta}{1-H}}\right)
$$

If $1-\alpha \beta\left[1-\frac{1-\beta}{1-H}\right]^{-1} \geq 0$, then $\mathrm{N}>0$ unambiguously. In what follows we consider the case in which $1-\alpha \beta\left[1-\frac{1-\beta}{1-H}\right]^{-1}$ is negative. Since an interior solution to the central planner's problem implies $\delta \theta^{\alpha}<1$ and the share $\phi^{l}<1$, we obtain a lower bound for (84) by eliminating $\delta \theta^{\alpha}$ and $\phi^{l}$ from the second term in (84)

$$
N_{1}>N_{2} \equiv\left(1-\frac{1-\beta}{1-\frac{H}{u}}\right)^{\alpha}+(1-u)\left(1-\frac{1-\beta}{1-H}\right)^{\alpha}\left(1-\frac{\alpha \beta}{1-\frac{1-\beta}{1-H}}\right)
$$

Because $\beta>\frac{H}{u}$, we have that $\beta-H>\beta(1-u)$. Thus,

$$
\begin{aligned}
N_{2}>N_{3} & \equiv\left(\frac{\beta-\frac{H}{u}}{1-\frac{H}{u}}\right)^{\alpha}+(1-u)\left(\frac{\beta(1-u)}{1-H}\right)^{\alpha}\left(1-\frac{\alpha \beta(1-H)}{\beta(1-u)}\right) \\
& =\left(\frac{\beta-\frac{H}{u}}{1-\frac{H}{u}}\right)^{\alpha}+(1-u)\left(\frac{\beta(1-u)}{1-H}\right)^{\alpha}-\alpha(1-H)\left(\frac{\beta(1-u)}{1-H}\right)^{\alpha} \\
& =\left(\frac{\beta-\frac{H}{u}}{1-\frac{H}{u}}\right)^{\alpha}+\left(\frac{\beta(1-u)}{1-H}\right)^{\alpha}[1-u-\alpha(1-H)] . \\
& =\left[\left(\frac{(1-H)\left(\beta-\frac{H}{u}\right)}{1-\frac{H}{u}}\right)^{\alpha}+(\beta(1-u))^{\alpha}[1-u-\alpha(1-H)]\right] \frac{1}{(1-H)^{\alpha}}
\end{aligned}
$$

Since $1-H>1-u$ and the first term in brackets is positive, it follows that

$$
N_{3}>N_{4} \equiv\left[\left(\frac{\beta-\frac{H}{u}}{\beta\left(1-\frac{H}{u}\right)}\right)^{\alpha}+1-u-\alpha(1-H)\right]\left[\frac{\beta(1-u)}{1-H}\right]^{\alpha} .
$$

Since $N_{4}<N_{3}<N_{2}<N$ and $\frac{\beta(1-u)}{1-H}>0$, we have that

$$
N>0 \quad \text { if } \quad\left(\frac{\beta-\frac{H}{u}}{\beta\left(1-\frac{H}{u}\right)}\right)^{\alpha}+1-u-\alpha(1-H)>0 .
$$


Since $\frac{\beta-\frac{H}{u}}{\beta\left(1-\frac{H}{u}\right)}<1$ and $\alpha \in(0,1)$, we have that $\left(\frac{\beta-\frac{H}{u}}{\beta\left(1-\frac{H}{u}\right)}\right)^{\alpha}>\frac{\beta-\frac{H}{u}}{\beta\left(1-\frac{H}{u}\right)}$. Therefore,

$$
N>0 \text { if } X>0, \quad \text { with } X \equiv \frac{1-\frac{H}{u \beta}}{1-\frac{H}{u}}+1-u-\alpha(1-H)>0 .
$$

If $\alpha=0$, then $X$ is unambiguosly positive. However, for $\alpha>0$ it is not clear what is the sign of $X$ for all admissible $(u, H, \beta)$. To determine the sign, we will derive a lower bound of $X$ by using the condition for existence of an RSE $\theta>\underline{\theta}$ and the condition for an interior solution to the central planner's problem $\delta \theta^{\alpha}<1$. The conditions $\theta>\underline{\theta}$ and $\delta \theta^{\alpha}<1$ imply $\delta \underline{\theta}^{\alpha}<1$ :

$$
\begin{aligned}
\delta \underline{\theta}^{\alpha} & =\frac{1}{u \beta}\left(1-\frac{H}{u}+H\right)\left(\frac{1-\beta}{1-\frac{H}{u}}\right)^{1-\alpha}<1 \\
& \Leftrightarrow \frac{1}{u \beta}\left(1-\frac{H}{u}+H\right)\left(\frac{1-\beta}{1-\frac{H}{u}}\right)<\left(\frac{1-\beta}{1-\frac{H}{u}}\right)^{\alpha} \\
& \Leftrightarrow-\frac{H}{u \beta}\left(\frac{1-\beta}{1-\frac{H}{u}}\right)>\frac{1-\beta}{u \beta}-\left(\frac{1-\beta}{1-\frac{H}{u}}\right)^{\alpha} \\
& \Leftrightarrow \frac{-\frac{H}{u \beta}}{1-\frac{H}{u}}>\frac{1}{u \beta}-\left(\frac{1-\beta}{1-\frac{H}{u}}\right)^{\alpha} \frac{1}{1-\beta}
\end{aligned}
$$

It follows that

$$
\Leftrightarrow \frac{1-\frac{H}{u \beta}}{1-\frac{H}{u}}>\frac{1}{u \beta}-\left(\frac{1-\beta}{1-\frac{H}{u}}\right)^{\alpha} \frac{1}{1-\beta}+\frac{1}{1-\frac{H}{u}} .
$$

Substituting (88) in (87), we derive the following lower bound for $X$.

$$
X>\underline{X} \equiv \frac{1}{u \beta}-\left(\frac{1-\beta}{1-\frac{H}{u}}\right)^{\alpha} \frac{1}{1-\beta}+\frac{1}{1-\frac{H}{u}}+1-u-\alpha(1-H) .
$$

Equipped with (89) we can prove that the inequality in (87) holds for all admissible $(\alpha, u, H, \beta)$. Let's consider 3 cases. First, if $\alpha=0$, (87) implies that

$$
X(\alpha=0)=\frac{1-\frac{H}{u \beta}}{1-\frac{H}{u}}+1-u>0 .
$$

Second, if $\alpha=1,(89)$ implies that

$$
X(\alpha=1)>\underline{X}(\alpha=1)=\frac{1}{u \beta}-\frac{1}{1-\frac{H}{u}}+\frac{1}{1-\frac{H}{u}}+1-u-(1-H)=\frac{1}{u \beta}+(1-u)-(1-H)>0 .
$$

Third, taking the derivative of $X$ with respect to $\alpha$

$$
\frac{\partial X}{\partial \alpha}=-(1-H)<0 \text { for all } \alpha \in(0,1) .
$$

The negative sign of $\frac{\partial X}{\partial \alpha}$ follows from parameter restriction (11). Since $X(\alpha=0)$ and $X(\alpha=1)$ are positive and $X$ is decreasing in $\alpha$, it follows that $X(\alpha)>0$ for all $\alpha \in[0,1]$. This implies that 
$N$, the numerator of (41), is positive. Since the denominator of (41) is positive for any $\theta^{a} \delta<1$, it follows that $W^{r}>0$ for all admissible $(\alpha, u, H, \beta)$

Proof of Proposition 6.1. Throughout we assume that $\theta>\underline{\theta}^{s}$ so that the returns condition (54) is satisfied. In the equilibrium of Proposition 6.1, all $\theta$-entrepreneurs issue standard bonds and never default. Meanwhile, $\varepsilon$-entrepreneurs default if $\varepsilon_{t+1}=0$. Thus, each $\theta$-entrepreneur expects next period a unique price $p_{t+1}$ and that a bailout will be granted if and only if $\varepsilon_{t+1}=0$. Given these expectations, a $\theta$-entrepreneur's problem is to choose whether to issue standard bonds or catastrophe bonds, and whether to implement a diversion scheme or not. We will show that if the bailout is not too generous, a $\theta$-entrepreneur has no incentives to deviate from the equilibrium.

First, if a $\theta$-entrepreneur issues standard bonds and will never default, her borrowing limit is $b_{t}^{s}=\left[m^{s}-1\right] w_{t}$ and and her expected profits are the same as those of the equilibrium safe plan of Proposition 3.1, given by (53). Second, consider no-diversion plans with catastrophe bonds that will not default. To break even, lenders require an interest rate no smaller than

$$
1+\rho^{c}=1 /[1-\lambda] \delta
$$

In a no-diversion plan lenders lend up to an amount that satisfies

$$
\delta[1-\lambda]\left[1+\rho^{c}\right] b_{t}^{c, n d} \leq h\left[w_{t}+b_{t}^{c, n d}\right] .
$$

Substituting (90) in the no-diversion condition (91) implies that the borrowing constraint with catastrophe bonds is $b_{t}^{c, n d} \leq \frac{H}{1-H} w_{t}$. Notice that this borrowing limit is the same as the one under the equilibrium strategy with standard debt: $b_{t}^{s}=\left[m^{s}-1\right] w_{t}$. Furthermore, the expected debt repayments are the same under both types of debt (i.e., $b_{t}^{s}\left[1+\rho^{s}\right]=b_{t}^{c, n d}[1-\lambda]\left[1+\rho^{c}\right]$ ). Thus, the expected profits are the same under both types of debt. Hence, conditional on no default, the $\theta$-entrepreneur has no incentives to deviate from the equilibrium of Proposition 6.1.

Third, consider plans where the $\theta$-entrepreneur issues catastrophe bonds and will default next period (in the $\varepsilon_{t+1}=0$ state). Since catastrophe bonds promise to repay only in the $\varepsilon_{t+1}=0$ state, these plans include both diversion plans and no-diversion plans with an excessive promised repayment that will make the firm insolvent. Under both plans, lenders are willing to lend up to the present value of the bailout that they will receive in the $\varepsilon_{t+1}=0$ state

$$
b_{t}^{c, d e f}=\delta[1-\lambda] \Gamma_{t+1}
$$

Since the bailout will be $\Gamma_{t+1}=\gamma y_{t+1}^{\theta}$, condition $\gamma<\bar{\gamma}^{\prime}$ in Proposition 6.1 implies that the 
borrowing limit for plans that lead to default is lower than the limit for non-defaulting plans

$$
\begin{aligned}
b_{t}^{c, d e f} & =\delta[1-\lambda] \gamma y_{t+1}^{\theta}<\left[m^{s}-1\right] w_{t}=b_{t}^{s} \\
& \Leftrightarrow \gamma<\bar{\gamma}^{\prime}=\frac{\left[m^{s}-1\right]}{\delta[1-\lambda]} \frac{w_{t}}{y_{t+1}^{\theta}}=\frac{\left[m^{s}-1\right]}{\delta[1-\lambda]} \frac{[1-\beta] \alpha}{1-\phi} \frac{1}{[\theta \phi]^{\alpha}}
\end{aligned}
$$

This bound is time-invariant because along the equilibrium path $\frac{w_{t}}{y_{t+1}^{\theta}}$ is constant:

$$
\frac{w_{t}}{y_{t+1}^{\theta}}=\frac{w_{t}}{y_{t}} \frac{y_{t}}{y_{t+1}^{\theta}}=\frac{(1-\beta) p_{t} q_{t}}{\frac{(1-\phi) p_{t} q_{t}}{\alpha}} \cdot \frac{1}{[\theta \phi]^{\alpha}}=\frac{[1-\beta] \alpha}{1-\phi} \cdot \frac{1}{[\theta \phi]^{\alpha}}
$$

Consider a no-diversion plan with catastrophe bonds that leads to default. Under such plan a $\theta$-entrepreneur borrows up to $b_{t}^{c, d e f}$, promises an interest rate $1 /[1-\lambda] \delta$, and will become insolvent if $\varepsilon_{t+1}=0$. Under this deviation a $\theta$-entrepreneur avoids repaying debt altogether, but it sacrifices profits in the $\varepsilon_{t+1}=0$ state. The requirement that the firm be insolvent in the $\varepsilon_{t+1}=0$ state, implies that the maximum payoff under this deviation is $\lambda \Gamma_{t+1}$ (because the highest revenue consistent with insolvency in the $\varepsilon_{t+1}=0$ state is $\left.b_{t}^{c, d e f}\left[1+\rho^{c}\right]=\Gamma_{t+1}\right)$.

$$
E \bar{\pi}_{t+1}^{c, d e f} \leq \lambda \Gamma_{t+1}=\lambda \gamma y_{t+1}^{\theta}=\lambda \gamma \frac{y_{t+1}^{\theta}}{y_{t}^{\theta}} y_{t}^{\theta}=\lambda \gamma \frac{y_{t}^{\theta}}{[\theta \phi]^{\alpha}}=\lambda \gamma \frac{1}{[\theta \phi]^{\alpha}} \frac{1-\phi}{[1-\beta] \alpha} w_{t}
$$

The last equality follows from (94). Comparing this upper bound with the equilibrium payoff in (53), we find that this deviation is not profitable provided the generosity of the guarantee is below $\bar{\gamma}^{\prime \prime}$

$$
\begin{aligned}
E \pi_{t+1}\left(b_{t}^{c, \text { def }}\right) & <\pi_{t+1}\left(b_{t}^{s}\right) \Longleftrightarrow \frac{\lambda \gamma}{[\theta \phi]^{\alpha}} \frac{[1-\phi] w_{t}}{[1-\beta] \alpha}<\left[\theta^{\alpha} \phi^{\alpha-1}-h\right] \frac{\phi w_{t}}{1-\beta} \\
& \Longleftrightarrow \gamma<\bar{\gamma}^{\prime \prime} \equiv \alpha\left[\theta^{\alpha} \phi^{\alpha-1}-h\right] \frac{\theta^{\alpha} \phi^{\alpha+1}}{1-\phi}
\end{aligned}
$$

Consider next diversion plans with catastrophe bonds. In a diversion plan the entrepreneur incurs a cost $h\left[w_{t}+b_{t}^{c}\right]$ at $t$, and is able to divert funds at $t+1$ provided she is solvent. Under such plan her borrowing limit is $b_{t}^{c, d e f}$ in (92). One can show that this deviation is not profitable because the debt ceiling under diversion is lower than under the equilibrium strategy $\left(b_{t}^{c, d e f}<b_{t}^{s}\right)$, which is implied by $\gamma<\bar{\gamma}^{\prime}$. In sum, $\theta$-entrepreneurs issue standard debt, do not divert and invest according to Proposition 3.1 if the bailout is not too generous: $\gamma<\min \left\{\bar{\gamma}^{\prime}, \bar{\gamma}^{\prime \prime}\right\}$.

Consider next the $\varepsilon$-entrepreneurs. Since the $\varepsilon$-technology has negative NPV, $\varepsilon$-agents find it profitable only to issue catastrophe bonds. In the presence of bailout guarantees, lenders are willing to buy these catastrophe bonds. Given the expected bailout $\Gamma_{i, t+1}$, lenders are willing to lend to each $\varepsilon$-agent up to an amount $b_{t}^{c}=\delta[1-\lambda] \Gamma_{t+1}$ at a rate $\rho_{t}^{c}$ (in (90)). At $t+1$, if the good state realizes $\left(\varepsilon_{t+1}=\bar{\varepsilon}\right)$, lenders will get zero-as promised-while if $\varepsilon_{t+1}=0$ lenders will get 
the bailout $\Gamma_{t}=b_{t}^{c}\left[1+\rho_{t}^{c}\right]$. It follows that an $\varepsilon$-agent will de-facto repay zero in all states of the world, and so he does not gain anything by implementing a diversion scheme. His expected payoff is $E \pi_{t+1}^{\varepsilon}=\lambda \bar{\varepsilon} b_{t}^{c}=\lambda \bar{\varepsilon} \delta[1-\lambda] \Gamma_{t+1}$. Since he does not need to risk his own capital, the $\varepsilon$-agent finds this project profitable.

Fiscal Solvency. Since the non-diverting part of the economy can be taxed in a lum-sum way, bailouts are financeable via domestic taxation provided

$$
E\left(\sum_{t=0}^{\infty} \delta^{t+1}\left[(1-\alpha) y_{t+1}^{\theta, n d}+\pi_{t+1}^{\theta, n d}+\pi_{t+1}^{\varepsilon, n d}\right]\right) \geq E\left(\sum_{t=0}^{\infty} \delta^{t+1} \Gamma_{t+1}\right)
$$

We will show that condition (96) holds if and only if $\gamma \leq \bar{\gamma}^{\prime \prime \prime}$, with $\bar{\gamma}^{\prime \prime \prime}$ defined by (99). Using the derivation of (39) and setting $\pi_{t+1}^{\varepsilon, n d}=y_{t+1}^{\varepsilon, n d}$, it follows that the LHS of (96) is equal to the discounted sum of T-production in the no-diverting part of the economy:

$$
E\left(\sum_{t=0}^{\infty} \delta^{t+1}\left[(1-\alpha) y_{t+1}^{\theta, n d}+\pi_{t+1}^{\theta, n d}+\pi_{t+1}^{\varepsilon, n d}\right]\right)=E\left(\sum_{t=0}^{\infty} \delta^{t+1}\left[y_{t+1}^{\theta, n d}+y_{t+1}^{\varepsilon, n d}\right]\right)
$$

Using (97) and the fact that a bailout occurs with probability $1-\lambda$, we can rewrite (96) as follows:

$$
E\left(\sum_{t=0}^{\infty} \delta^{t+1}\left[y_{t+1}^{\theta, n d}+y_{t+1}^{\varepsilon, n d}\right]\right) \geq \sum_{t=0}^{\infty} \delta^{t+1} \gamma[1-\lambda] y_{t+1}^{\theta, n d}
$$

Since bailouts are granted only in the $\varepsilon_{t+1}=0$ state, and in this state all $\theta$-firms are solvent, while all $\varepsilon$-firms go bust $\left(y_{t+1}^{\varepsilon, n d}=\varepsilon_{t+1} I_{t}^{\varepsilon}=0\right)$, the bailout payment if $\varepsilon_{t+1}=0$ is $\Gamma_{t+1}=\gamma y_{t+1}^{\theta, n d}$. Therefore, (98) can be re-expressed as:

$$
\begin{aligned}
\sum_{t=0}^{\infty} \delta^{t+1} y_{t+1}^{\theta, n d}[1+\lambda \bar{\varepsilon} \delta[1-\lambda] \gamma-\gamma[1-\lambda]] & \geq 0 \\
\sum_{t=0}^{\infty} \delta^{t+1} y_{t+1}^{\theta, n d}[1+\gamma[1-\lambda][\lambda \bar{\varepsilon} \delta-1]] & \geq 0 \\
\frac{y_{o}^{s}}{1-\delta\left(\theta \phi^{s}\right)^{\alpha}}[1+\gamma[1-\lambda][\lambda \bar{\varepsilon} \delta-1]] & \geq 0 \quad \text { if } \delta\left(\theta \phi^{s}\right)^{\alpha}<1 \\
\frac{\left(1-\phi^{s}\right)^{\alpha}}{1-\delta\left(\theta \phi^{s}\right)^{\alpha}} q_{o}^{\alpha}[1+\gamma[1-\lambda][\lambda \bar{\varepsilon} \delta-1]] & \geq 0 \Leftrightarrow \gamma \leq \bar{\gamma}^{\prime \prime \prime} \equiv \frac{1}{[1-\lambda][1-\lambda \bar{\varepsilon} \delta]}
\end{aligned}
$$

Since $\phi^{s}<1$ and $\delta\left(\theta \phi^{s}\right)^{\alpha}<1$, the LHS is non-negative iff $\gamma \leq \bar{\gamma}^{\prime \prime \prime}$. Putting together the three bounds in (93), (95) and (99) we conclude that the equilibrium of Proposition 6.1 exists if $\gamma \leq \bar{\gamma}$, with

$$
\bar{\gamma} \equiv \max \left\{\frac{h \alpha}{[1-\lambda]} \frac{\phi}{1-\phi} \frac{1}{[\theta \phi]^{\alpha}}, \alpha\left[\theta^{\alpha} \phi^{\alpha-1}-h\right] \frac{\theta^{\alpha} \phi^{\alpha+1}}{1-\phi}, \frac{1}{[1-\lambda][1-\lambda \bar{\varepsilon} \delta]}\right\}, \quad \phi=\phi^{s}=\frac{1-\beta}{1-H}
$$

